\title{
Performance Characterization of Hitachi HD-2300A STEM with Dual-EDS Configuration
}

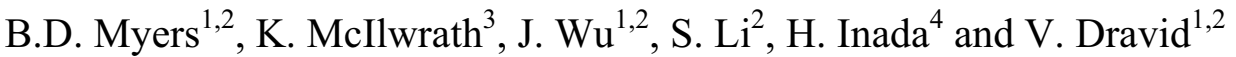

1. Department of Materials Science and Engineering, Northwestern University, 2220 Campus Drive, Evanston, IL 60208

2. NUANCE Center, Northwestern University, 2220 Campus Drive, Evanston, IL 60208

3. Hitachi High Technologies America, Inc., 5100 Franklin Drive, Pleasanton, CA 94588

4. Hitachi High-Technologies Corp. 882 Ichige Hitachinaka, Ibaraki 312-8504, Japan

Electron microscopy in biological systems has traditionally been focused on "structure", which is often analyzed by imaging and 3-D tomography with diverse modalities (e.g., TEM, STEM ADF/HAADF, EFTEM). In several classes of biological structures and phenomena, the role of local chemistry, particularly of metals (e.g., $\mathrm{Zn}, \mathrm{Cu}, \mathrm{Se}$ ), has emerged as an important consideration which holds the key for unraveling important signal transduction pathways. However, the problem of detection and spatial variation of chemical species in biological systems is further exasperated by their minute amounts (other than the usual suspects; i.e., low atomic number elements such as, $\mathrm{C}, \mathrm{H}$, $\mathrm{O}, \mathrm{N}$ ) and the ubiquitous radiation damage which limits the analytical sensitivity due to dosage considerations. EELS provides an excellent approach for chemical analysis of many elements (e.g., $\mathrm{Ca}, \mathrm{Cu}, \mathrm{Fe}$ ), but for other elements such as $\mathrm{Zn}, \mathrm{P}$ and Se the cross-section for EELS is poor and EDS is a more desirable technique. Recognizing the need for analytical studies in biology, we have designed and developed a new dedicated STEM instrument, based on the commercial Hitachi HD$2300 \mathrm{~A}$ scanning transmission electron microscope (STEM). The newly designed HD-2300A is equipped with two $\mathrm{Si}(\mathrm{Li})$ EDS detectors from Thermo-Fisher for a combined nominal solid angle of $\sim 0.76 \mathrm{Sr}(\sim 0.38 \mathrm{Sr}$ each $)$, to increase EDS analytical sensitivity. Here, we present salient features and performance characteristics of the novel dual-EDS Hitachi HD-2300A STEM.

The standard $\mathrm{NiO}$ test specimen approach by Bennett and Egerton was utilized for EDS characterization, as summarized in Table 1 [1]. Since the two EDS detectors are mounted on opposite sides of the column, all tests were performed near the center of the grid with $0^{\circ}$ sample tilt and a specially designed dual cut-out low background holder. A detail view of the Ni-K peaks from a $200 \mathrm{~s}$ acquisition at $200 \mathrm{kV}$ is shown in Figure 1. Film count ratio (FCR) data was collected to assess the influence of hard X-rays and scattered electrons generated in the microscope column. The FCR data was collected with the beam scanning a $\sim 90 \times 90 \mathrm{~nm}$ region in the center of a grid square for 80 , 120 and $200 \mathrm{kV}$. In addition to FCR, hole count ratio (HCR) measurements performed using the same $\mathrm{NiO}$ test sample in a grid square with no film. Further, the peak-to-background was measured according to the Fiori method $\left(\mathrm{P} / \mathrm{B}_{10}\right)$ as plotted in Figure 2 [2]. The system exhibits very low stray radiation due, in part, to a thick $(1.0 \mathrm{~mm}) \mathrm{Pt}$ EDS aperture.

Overall, as the preceding data show, the performance of the EDS system is excellent and provides the desired increase in collection efficiency. It should be noted that our dual-EDS system utilizes Be windows to obtain the desired high solid angle as ultra-thin polymer windows do not allow maximal collection efficiency due to Si grid-bars. In addition, the electron optics in the HD$2300 \mathrm{~A}$ were modified from the standard configuration in order to accommodate the dual-EDS system and high solid angle. As such, the imaging performance of the system was tested as well and found to exhibit only a minor compromise from the standard lens design. Figure 3 shows a high resolution bright field STEM image of a FIB prepared Si sample oriented along the [110] zone axis 
and the corresponding high-angle annular dark field image (HAADF). The presentation will cover various aspects of design optimization for dual-EDS STEM and corresponding analytical STEM applications to materials and biology problems anchored by high analytical sensitivity.

References

[1] J.C. Bennett and R.F. Egerton, Microscopy and Microanalysis. 1:4 (1995) 143.

[2] C.E. Fiori et al. Microbeam Analysis ed. by K.F.J. Heinrich, San Francisco Press, San Francisco 1982.

[3] This work was performed in the EPIC facility of NUANCE Center at Northwestern University. Research was supported by the W. M. Keck Foundation Medical Research Award.

TABLE 1. EDS performance characterization data from $\mathrm{NiO}$ test specimen.

\begin{tabular}{|c|c|c|c|c|c|c|c|c|c|}
\hline \multirow{2}{*}{$\begin{array}{c}\text { Beam Energy } \\
\text { (keV) }\end{array}$} & \multicolumn{3}{|c|}{ Film Count Ratio (FCR) } & \multicolumn{3}{c|}{ Hole Count Ratio (HCR) } & \multicolumn{3}{c|}{ Fiori Number (P/B (10 $_{\text {) }}$} \\
\cline { 2 - 10 } & Det 1 & Det 2 & Both & Det 1 & Det 2 & Both & Det 1 & Det 2 & Both \\
\hline $\mathbf{8 0}$ & 7.8 & 8.7 & 7.6 & 1178 & 1434 & 1609 & 1920 & 2590 & 2108 \\
\hline $\mathbf{1 2 0}$ & 9.7 & 8.0 & 8.1 & 321 & 291 & 727 & 3172 & 3015 & 2774 \\
\hline $\mathbf{2 0 0}$ & 13.0 & 12.2 & 11.8 & 91 & 88 & 91 & 2248 & 3070 & 2446 \\
\hline
\end{tabular}

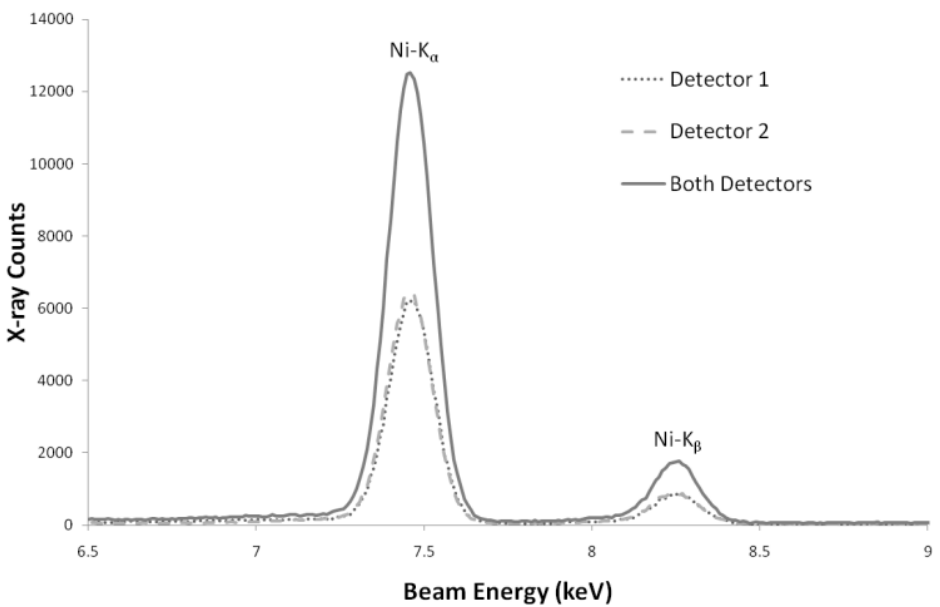

FIG. 1. Detail view of spectra collected for 200s at $200 \mathrm{keV}$ from $\mathrm{NiO}$ test specimen.
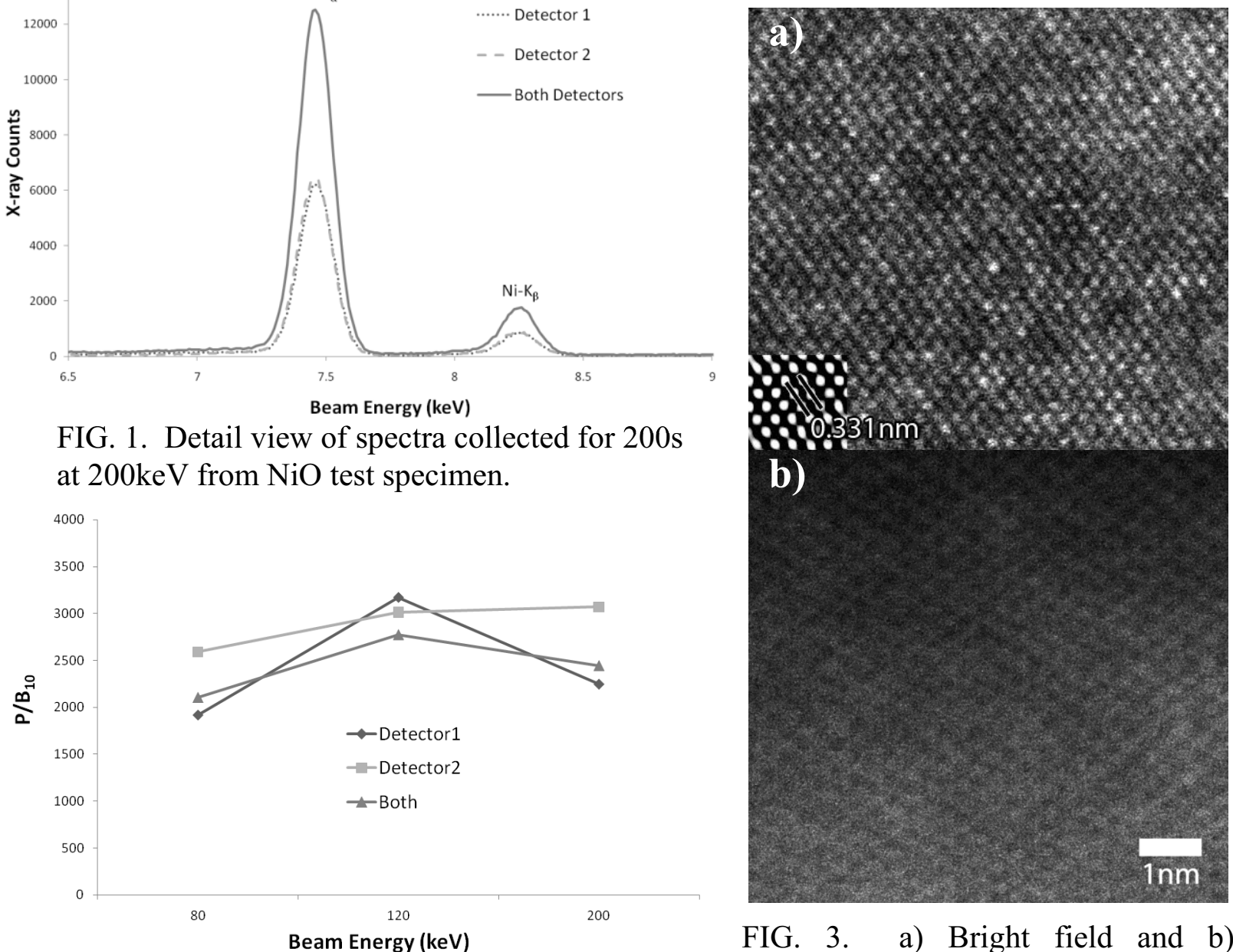

FIG. 2. Fiori numbers $\left(\mathrm{P} / \mathrm{B}_{10}\right)$ for $\mathrm{NiO}$ test specimen at 80,120 and $200 \mathrm{keV}$.

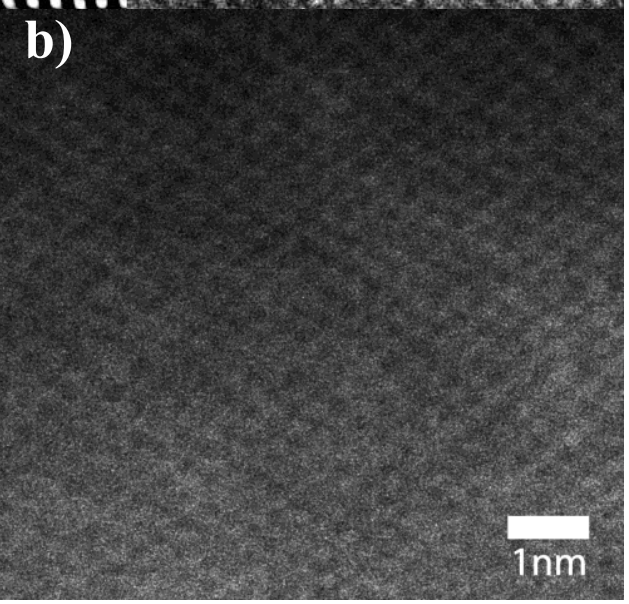

FIG. 3. a) Bright field and b) HAADF STEM images of $\mathrm{Si}$ along [110] zone. 